\title{
Dynamics of the Net Systems, Capturing Space Debris
}

\author{
By Pavel M. TrivaILO ${ }^{1)}$ and Hirohisa KoJIMA ${ }^{2)}$ \\ ${ }^{1)}$ School of Aerospace, Mechanical and Manufacturing Engineering, RMIT University, Melbourne, Australia \\ ${ }^{2)}$ Department of Aerospace Engineering, Tokyo Metropolitan University, Tokyo, Japan
}

(Received July 31st, 2015)

\begin{abstract}
According to NASA, more than 20,000 pieces of debris are tracked as they orbit the Earth. They all travel at speeds up to 7,800 $\mathrm{m} / \mathrm{s}$, fast enough for a relatively small piece of orbital debris to damage a space vehicle, International Space Station or any future space system. Therefore, research on the space debris removal and mitigation of the impact of the space debris is attracting more and more attention of the international space community, including engineers, scientists, designers and space experts. In the current paper, the comprehensive non-linear models of following net capturing systems for debris removal are developed: (a) a rapidly deployed system, involving spider-like webs, ejected towards the remote targeted debris; (b) spin-stabilised ejected spider-like webs, (c) a framed static net system for capturing the on-going debris. Various simulated demonstration cases include symmetric and asymmetric (off-set) collisions of the ejected webs with the single and multiple obstacles, collision of the single and multiple debris with the stationary webs, cases of significant impacts, leading to the failure/rupture of the web. In all cases we were able to assess the dynamical parameters of the system and observe the influence of various operational parameters on the dynamics of the post-collision processes. These competing models are then used to study dynamics of the deployment of the net systems and their interaction with the capturing objects. A special attention is given to the biologically inspired nets, resembling with their shapes the spider webs. The dynamic analysis is complemented with the analysis of the stresses in the webs, used in various designs. Various study cases are illustrated with the animations of the representative cases in Virtual Reality.
\end{abstract}

Key Words: Space Debris, Capture, Multiple Impact, Spider Orb Web Geometry, Finite Element Method (FEM)

\section{Nomenclature}

$\begin{array}{cll}c & : & \text { web's viscous damping coefficient }[\mathrm{N} \cdot \mathrm{s} / \mathrm{m}] \\ k & : & \text { axial stiffness of the web's thread }[\mathrm{N} / \mathrm{m}] \\ L & : & \text { total length of web's threads }[\mathrm{N} / \mathrm{m}] \\ L_{\max } & : & \text { maximal allowd length of finite element }[\mathrm{m}] \\ M_{e} & : & \text { web's ray end mass }[\mathrm{kg}] \\ N_{F E} & : & \text { number of finite elements }(\mathrm{FE}) \\ N_{N} & : & \text { number of nodes in the FEM model } \\ N_{r} & : & \text { number of radii } \\ N_{s} & : & \text { number of spirals } \\ R & : & \text { outer radius of the web [m] } \\ s & : & \text { length of the arc of the spirals }[\mathrm{m}] \\ v & : & \text { velocity of the web (m/s) } \\ \alpha & : & \text { constant of the arithmetic spiral } \\ t & : & \text { time }[\mathrm{s}] \\ \theta & : & \text { angle of the arithmetic spiral }\end{array}$

Subscripts

$\begin{array}{lll}0 & : & \text { initial } \\ \mathrm{f} & : & \text { final } \\ r & : & \text { radial } \\ s & : & \text { spiral }\end{array}$

\section{Introduction}

According to NASA, more than 500,000 pieces of debris are orbiting the Earth with approximately 20,000 of them are actually tracked. ${ }^{1)}$ They all travel at speeds up to $7,800 \mathrm{~m} / \mathrm{s}$ and present multiple serious threats for the human activities in space, their sustainability and restrict access to space.

The size of debris is approximately $5 \mathrm{~cm}$ to $10 \mathrm{~cm}$ in low
Earth orbit (LEO) and $30 \mathrm{~cm}$ to $1 \mathrm{~m}$ at geostationary altitudes (GEO). Only $6 \%$ of the catalogued orbit population is operational spacecraft, while $28 \%$ can be attributed to decommissioned satellites, spent upper stages, and mission related objects (launch adapters, lens covers, etc.).

Over $90 \%$ of the mass of debris in LEO is in 1,200 objects weighing more than $500 \mathrm{~kg}$.

The high impact velocities, which can reach $15 \mathrm{~km} / \mathrm{s}$ for most missions in LEO, are the reason for the destructive energy, even despite of the small object sizes. ${ }^{2)}$

Studies show that for a continued use of LEO, 5-10 large and strategically chosen debris need to be removed every year. To capture and de-orbit a large piece of defunct space hardware is no small challenge, both technically, legally and financially. ${ }^{3)}$ The importance of the problem has been articulated by scien-

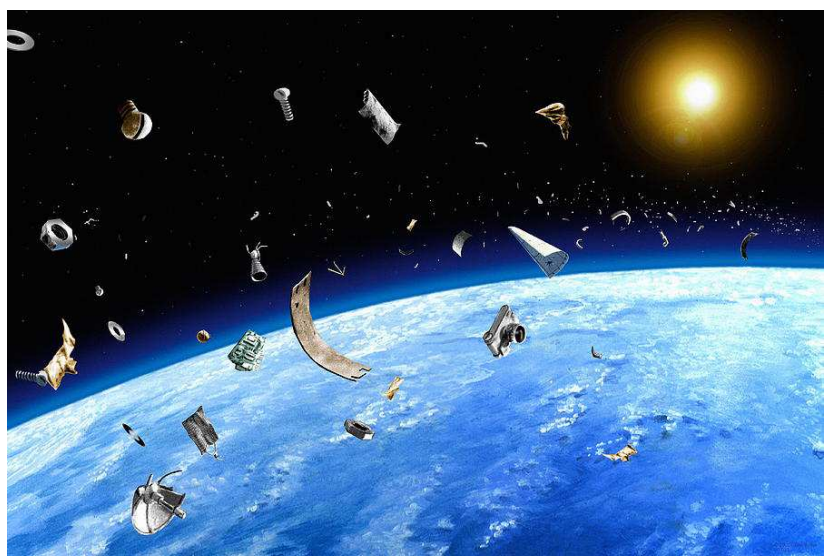

Fig. 1. Space Junk: A Future Menace (Courtesy: earthuntouched.com). 
tists, media and artists (as shown in Fig. 1), it has also resulted in the rapidly increasing number of research publications, technical reports on the topic, experimental studies and attempts to test new technologies during real missions.

Various new analysis studies, engineering concepts, methods and mechanisms to solve the problem of space debris and tackle the challenges, associated with the space debris, have been recently proposed. The examples of the designs belong to various classes (mechanical, electrical, propulsion, etc.) and their combinations. Examples of the mechanical systems include tethered remote access systems, ${ }^{4) 5)}$ net-based capture systems, ${ }^{6)}$ 7) robotic arm/tentacles and harpoon systems. ${ }^{8}$

In the most studies, dedicated to the debris access/capture/removal, the use of flexible tethers or webs have been widely proposed and studied.

One of the most known and referred cases of using strong flexible systems in space is the TSS-1R mission. ${ }^{9)}$ It has employed the flexible tether with the following characteristics: outer diameter $2.54 \mathrm{~mm}$, deployed length $20.7 \mathrm{~km}$, breakstrength $1,780 \mathrm{~N}$, maximum allowable tension $980 \mathrm{~N}$, maximum expected load $100 \mathrm{~N}$, maximum allowable mass $8.2 \mathrm{~g} / \mathrm{m}$, temperature range -100 to $+125^{\circ} \mathrm{C}$.

In 1999, on-orbit satellite capture experiments have been carried out successfully by the ETS-VII satellite. ${ }^{10)}$ Pearson et al. introduced a low-cost solution for LEO space debris removal which is called Electro Dynamic Debris Eliminator (EDDE). This kind of capture uses lightweight expendable nets and realtime man-in-the-loop control. Authors claimed that "EDDE can affordably remove nearly all the 2,465 objects of more than $2 \mathrm{~kg}$ that are now in 500-2,000 km orbits". ${ }^{11)}$ The European Space Agency (ESA) has performed several system studies for orbital servicing, such as ROGER, Conexpress, or MART-OLEV for servicing GEO satellites, as well as CDF studies on active debris removal of large objects. ESA is studying the option of using a tethered capture system for controlled de-orbitation through pulling where the capture is performed using thrownets or alternatively a harpoon. ${ }^{2}$

The authors and colleagues have proposed various concepts of using long controlled flexible tethers for payloads and debris capture. ${ }^{4) 5) 12)-15)}$

Trivailo et.al ${ }^{13)}{ }^{14)}$ also reported about the proposal to use (for the capture of debris and their following management) the coneshaped web with the space propulsive system, shown conceptually in Fig. 2.

Carroll has focused on the risks of debris collision with the ISS, shuttle, or high-value satellites. ${ }^{16)}$ Carroll ${ }^{16)}$ proposed a system with 10 meter flight net (shown in Fig. 3) made of Spectra, weighing 50 grams, including suspension lines, one-shot rip-stop type post-capture energy absorbers, and a very small and light storage spool (attached to the center of the net, to prevent generation of loose debris).

Yi et al. ${ }^{17)}$ have reported about the design of the analysis of the triangle-mesh, square-mesh and hexagon-mesh webs.

Zhai et al. studied the kinematics and dynamics of a tethernet debris capture system. It is demonstrated that a flexible capture is better than a rigid on physical characteristics. ${ }^{18)}$ Benvenuto et al. ${ }^{7)}$ reported research dedicated to the design, analysis and test of a space debris capture net, shut from an active satellite. They discussed the effectiveness of a planar net ver-

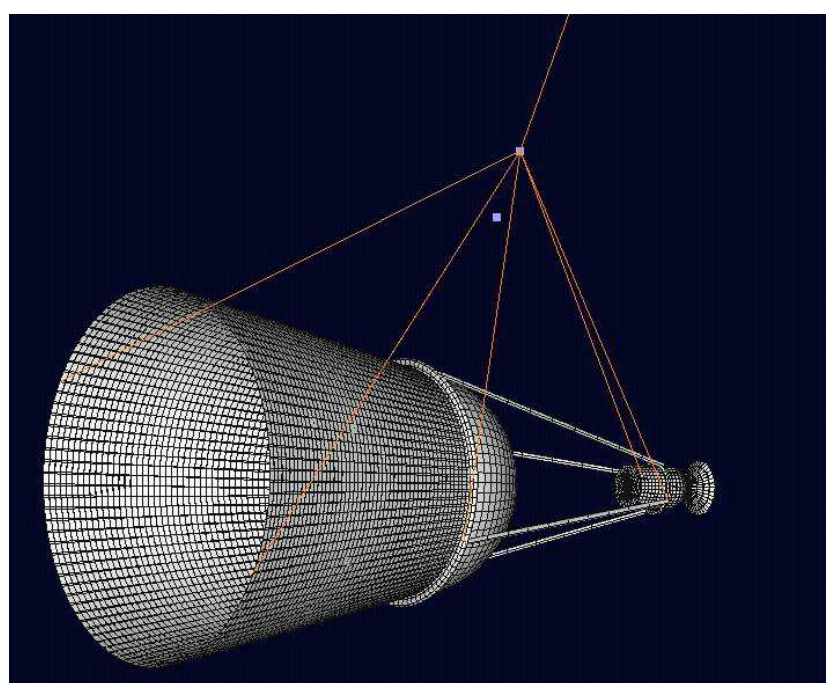

Fig. 2. Space web debris capturing system with rocket attached.

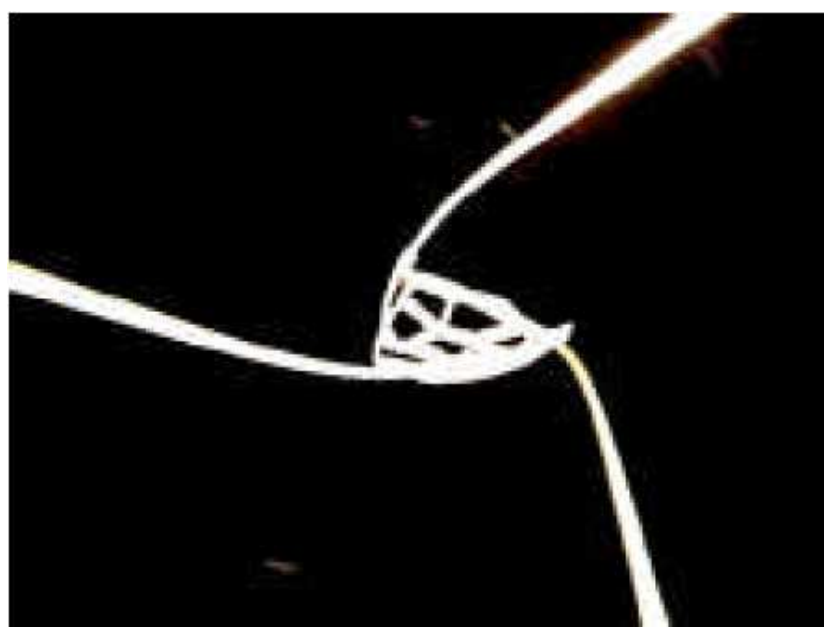

Fig. 3. Spin-stabilised capture nets.

sus a 3D (either conical and pyramidal shape) net configuration solutions according to the size, mass and configuration of the class of targets to be wrapped. Yang and colleagues developed a numerical method to calculate the static and dynamic response of triangle-mesh, square-mesh and hexagon-mesh webs under different conditions, and the mechanical characteristics of webs with the three meshes in the impact process were obtained. ${ }^{17)}$

In view of the importance of the strength considerations of the debris management systems, many researchers are trying to improve their designs, learning from the nature. Recently, various special studies unlocked the secrets of the high strength of the spider webs. Aoyanagi et al. ${ }^{19)}$ proposes a simple model to describe spider orb webs, and demonstrated that is the radial threads are sufficiently strong compared to the spiral threads, the model is free of stress concentrations even when a few spiral threads are broken, which is in contrast with what occurs in common elastic materials. Despite of the simple linear elastic characteristic of the web model, it was possible to demonstrate that an appropriately large value of the ratio $K / k$ between the radial $K$ and spiral $k$ stiffnesses of the (as in real spider webs) helps enhance the damage tolerance of the web.

Cranford at al. ${ }^{20)}$ have simulated spider webs, introducing sophisticated non-linear model of the material of the spider silks and involving four distinct regimes characteristics of the silk: 
(I) stiff initial response governed by homogeneous stretching; (II) entropic unfolding of semi-amorphous protein domains; (III) stiffening regime as molecules align and load is transferred to the b-sheet crystals; and (IV) stick-slip deformation of $\beta$ sheet crystals until failure. The numerical simulations by the authors confirmed that a nonlinear stress response results in superior resistance to structural defects in the web compared to linear elastic or elastic-plastic (softening) material behaviour.

This led to the statement that the superior performance of silk in webs is therefore not due merely to its exceptional ultimate strength and strain, but arises from the nonlinear response of silk threads to strain and their geometrical arrangement in a web. As a result, removal of up to $10 \%$ of threads, at different locations relative to the load, had little impact on the webs response; in fact, the ultimate load capacity increased by $310 \%$ with the introduction of defects.

Despite of the sophistication in the material models, the paper lacks the fidelity of the geometry of the real spider orb webs, as their webs are modeled as arithmetic spirals, supported by radial threads at regular intervals of $45^{\circ}$, whereas the radii number in the real orb-weaving spider webs is at the order of 32-36 ${ }^{21)}$ or 26-35, ${ }^{22)}$ as shown in Fig. 4 (a).

We also believe in high efficiency of the orb web spider nets

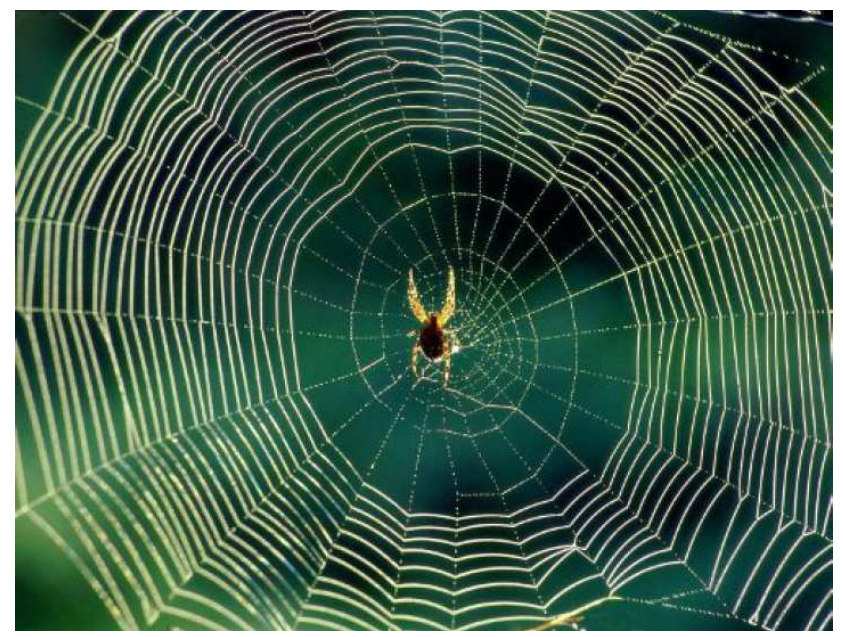

(a)

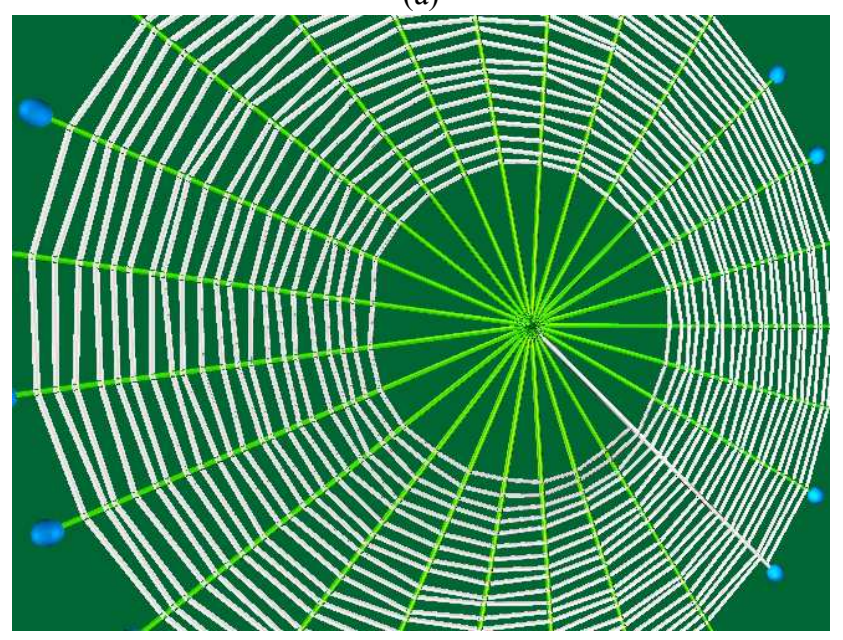

(b)

Fig. 4. Spiral webs: (a) real spider orb-web, ${ }^{22}$ (b) replicated artificial biolgically inspired "spider orb-web" net. (they are remarkably strong and light, damage tolerant and recyclable, possessing high energy absorption and ) and would like to apply the nature's evolutionary wisdom to the new space systems. We believe that there are many more hidden secrets in the spider webs, therefore would like to explore their unique dynamic performance properties and to apply them to the space capturing systems. So, in this paper, we also intend to promote the "biologically-inspired" approach to the design of the capturing devices, proposing: (1) to use the spider shaped nets for capturing the debris in space, (2) to analyse the capture of the single and multiple debris with the spider-type webs, (3) to understand the basics of the failure (rupture) of the space systems in order to assess the "re-usability" capacity of the webs to be used more than once. The re-usability would, probably, be one of most critical requirements for the space debris systems, as there the debris removing system should not potentially create more debris.

\section{Modelling of the Web's Geometries and Functions}

\subsection{Learning from the nature}

An example of the photo of the real spider orb-web, taken from natural landscapes, is shown in Fig. 4 (a). ${ }^{22)}$ Spider webs are complex nets of strong dragline threads (frame, spokes) radiating out from the center; and elastic, sticky catching threads spiraling into the center. An orb-weaver begins its web with radial and framework threads using dragline silk, providing a foundation upon which to spiral the sticky catching threads. The spiders then create an auxiliary spiral to help the radial threads support the spiders weight as it builds. Next, the spider uses, and subsequently destroys, the auxiliary spiral as a guide to create the catching spiral, which it dots with glue. What is perhaps the most amazing part of this hour-long process is that orb-weaving spiders often have poor eyesight and weave using only their sense of touch. ${ }^{23)}$

Similar to spiders, we start construction of the web(s) with the establishment of the initially selected $N_{r}$ radial threads. The typical number of radii number $N_{r}$ reported in the literature is approximately between 26 and 36 . It should be emphasised that radial threads are strong and the success of the high kinetic energy absorption of the spider webs is attributed to these ray

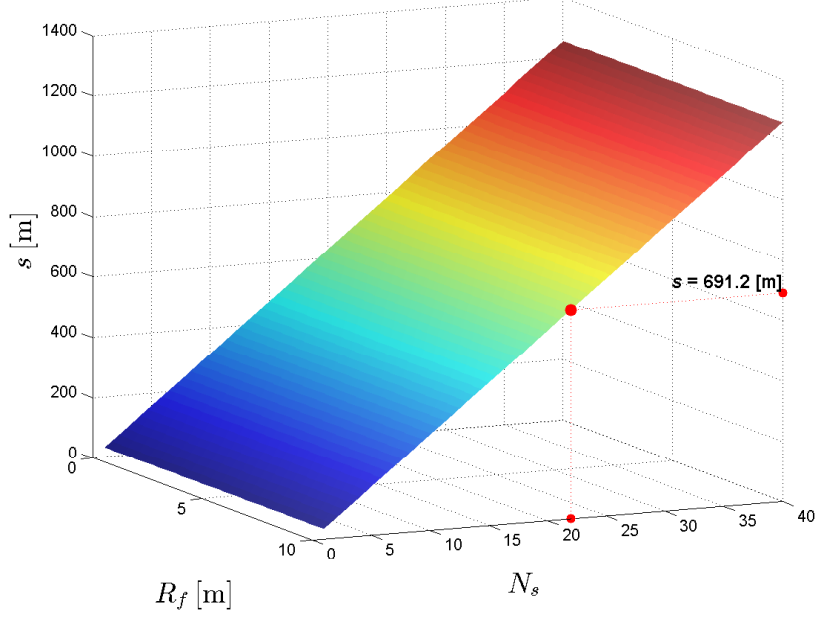

Fig. 5. Length of the spiral in the "spider orb-web" net. 
components of the webs. ${ }^{24)}$ At the top of the radial skeleton, the circumferential components must be added. It would be important to mention that radii are approximately three orders of magnitude stiffer than capture spirals and are therefore stretched to much lower extensions during most impacts. ${ }^{24)}$

Various previous models have reported concentric models, with perfect or slightly distorted circles. ${ }^{24) 25)}$ In our study we aim to be realistic in reproducing the web pattern and follow the method described by Cranford et al., ${ }^{20)}$ in which the orb web is approximated by an Archimedean or arithmetic spiral:

$$
R=\alpha \times \theta
$$

The length of the exact spiral can be calculated using Eq. (2):

$$
\begin{aligned}
s(\theta) & =\frac{1}{2} \alpha\left(\theta \sqrt{1+\theta^{2}}+\sinh ^{-1} \theta\right)= \\
& =\frac{1}{2} \alpha\left[\theta \sqrt{1+\theta^{2}}+\ln \left(\theta+\sqrt{1+\theta^{2}}\right)\right] .
\end{aligned}
$$

and can be used to estimate the amount material required to produce the spiral and also to estimate the weight of the web: for the real webs it would be overestimated value, as the spiral thread segments are rather straight, than follow the based on the classical Archimedean spiral shape.

Despite of the complex appearance of the Eq. (2), when it is used to produce a 3D plot for the $s$ as a function of $N_{r}$ and $R_{f}$, the shape of the plot (see Fig. 5) is not complex.

If the large (space dimensions) Archimedean spiral shape (web starting from origin and having $N_{r}=25$ and $N_{s}=22$, $R_{f}=10 \mathrm{~m}$ ) was produces, its length would be $691 \mathrm{~m}$.

For more flexibility, however, the spiral thread can be started at the initial radius $R_{0}$ :

$$
R(\theta)=R_{0}+\alpha \theta
$$

If we denote the number of full spirals as $N$ and the final radius of the web as $R_{f}=R_{0}+\alpha\left(2 \pi N_{r}\right)$, then we could get the following useful geometric relationship:

$$
\alpha=\left(R_{f}-R_{0}\right) /\left(2 \pi N_{r}\right)
$$

A dedicated MATLAB computer program has been designed to produce the nets, resembling the real spider's web geometries. In addition to the arbitrary choice of $N_{r}, N_{s}, R_{0}$ and $R_{f}$, it also enables to distort the perfect shape using the randomly generated factors. An example of the synthesised web is shown in Fig. 4 (b). In this case the deliberate choice of $N_{r}=25, N_{s}=20$ was prompted by the real spider web, shown in Fig. 4 (a).

\subsection{Finite element geometrical models}

The geometry of the designed web is modelled using MATLAB. There are two major stages in the process. Firstly, the base geometry is captured, as per the process outlined above. Then, the initial FEM mesh refined for higher fidelity results. An example of the biologically inspired web has $N_{r}=29$ radii and $N_{s}=26$ spirals. If we further assume $R_{0} / R=0.1$, $R_{f} / R=0.94$, this would result in the initial FEM mesh with 1,538 finite elements and 785 nodes. For higher accuracy of the simulations, this mesh can be refined with indtroduction of the the requirement for the maximum length of any element not to exceed $L_{\max }=0.25 \mathrm{~m}$. A refined web, having $N_{F E}=3,949 \mathrm{fi}-$ nite elements, and $N_{N}=3,196$ nodes is shown in Fig. 6 . The $\alpha$ parameter for the web can be calculated using Eq. (4), giving its value of 0.94 . The total length of the spiral thread, determined with Eq. (2) is equal to $L_{s}=686 \mathrm{~m}$, which is closely matching the actual value of $683 \mathrm{~m}$. In practical cases, when modelling of the $3 \mathrm{D}$ conical webs is needed, the out-of-plane coordinates for the nodes can be further assigned, as illustrated in one of the following sections.

\subsection{Major capabilities of the simulation package}

The authors developed a computer package to simulate various scenarios of using webs to capture single and mutiple flying objects, with the major motivation of its applications to space debris capture. It has versatile settings and features, with some of them being demonstrated in the following section. For brevity, we name here only its most significant features of the package:

(1) It enables generation of webs of various geometries: 2D and $3 \mathrm{D}$, spiral and concentric.

(2) It enables simulations of various scenarios, including: free flying rotating and non-rotating webs, colliding with sta-

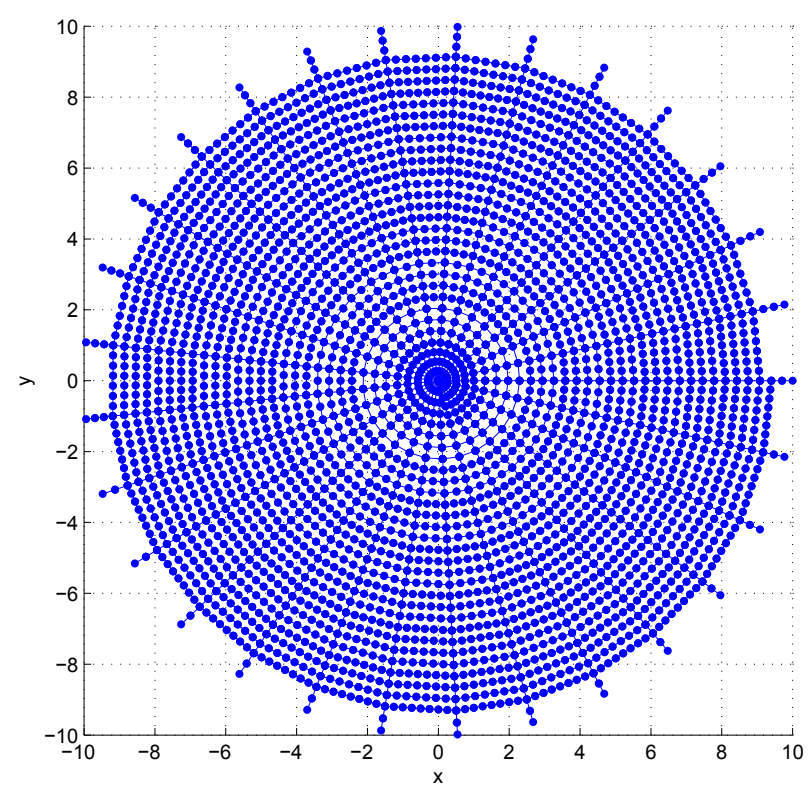

Fig. 6. Example of FEM model of the biologically inspired net.

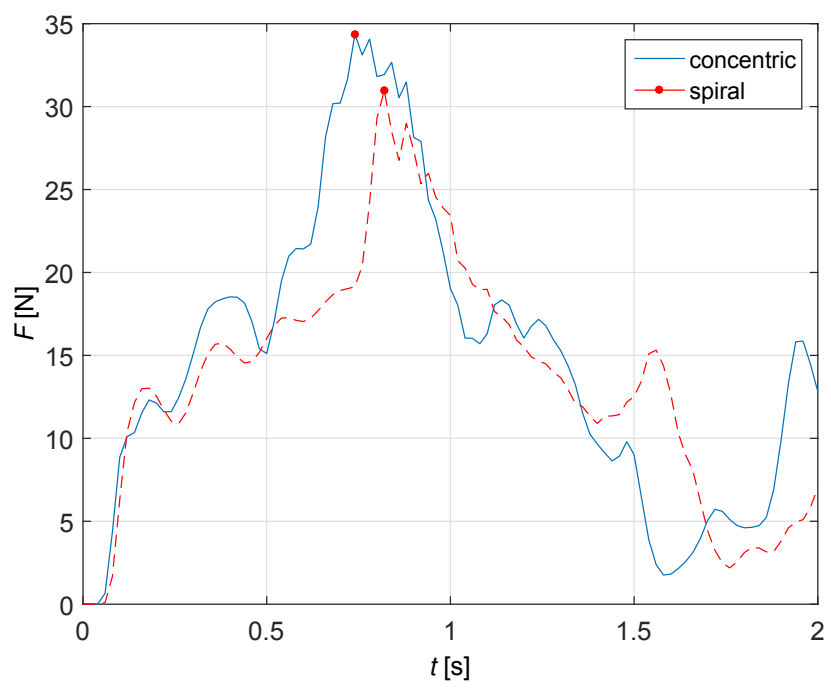

Fig. 7. Comparative time histories of the stresses in the most stressed elements of the spiral and concentric webs. 
tionary or moving capturing objects, and stationary webs with fixed boundaries, colliding with moving objects.

(3) The package can simulate interaction of the webs with colliding single or multiple objects, including spheres, cubes, cones, prisms or their combination.

(4) The package enabling modelling of a range of various friction models of interaction between the web and obstacles, with no-friction and stick-friction being extreme cases.

(5) The computer programs also enables monitoring of the time history of the stresses at various points of the web. In addition to this, it is possible to model the rupture of the selected threads in the web at the instants when the strength criteria are not met or as per predetermined scenarios.

(6) The package enables variation in all major parameters of the simulation cases, allowing design and optimisation of thegeometrical and physical parameters of the web system for its better performance.

(7) At last, the package enables visualisation of results in Virtual Reality.

\section{Capturing Webs Interacting with Captured Debris}

\subsection{Comparison test: spiral web versus concentric web}

Of course, it would be so appealing to apply the wisdom of spider orb-webs to the artificial space debris capturing systems. However, as the main secret of the amazing strength properties of the spider webs is in the non-linear properties of the web threads, full potentials of the sipder configurations could only be utilised when the scientists develop materials with similar non-linear properties.

Still, we can exploit the other benefits of the capturing webs, offered by the spiral geometrices. Firstly, as it appears, the spiral webs could be easier manufactured, as would have only one circumferencial component. Let us put for the simple test dynamics performance of two similar webs, spiral and concentric. As an illustrative exmple, let us first consider a web configuration, presented in Fig. 6 with fixed outer radial points and apply an axial impact load to the web at its centre with a cylinder of radius $0.3 R$. For completeness, we present here all major parameters of the case: $N_{r}=29, N_{s}=27, R_{0} / R=0.10, R_{f} / R=0.94$, $L=1,137.88[\mathrm{~m}], N_{F E}=1552, N_{N}=799, v=4[\mathrm{~m} / \mathrm{s}], \omega=0[\mathrm{rad} / \mathrm{s}]$, $m_{r}=0.03[\mathrm{~kg} / \mathrm{m}], m_{s}=0.02[\mathrm{~kg} / \mathrm{m}], c_{r}=10[\mathrm{~N} \cdot \mathrm{s} / \mathrm{m}], c_{s}=8[\mathrm{~N} \cdot \mathrm{s} / \mathrm{m}]$, $k_{r}=1,000[\mathrm{~N} / \mathrm{m}], k_{s}=500[\mathrm{~N} / \mathrm{m}]$. The responses of the spiral and concentric webs were similar: the associated maximum values of the forces in both cases were experienced in the outer radial segments. However, as it can be evidenced by Fig. 7, the value of the maximum force $F_{1 \text { max }}=30.98 \mathrm{~N}$ in the spiral element was slightly smaller, than the maximum value of the force $F_{2 \max }=34.36 \mathrm{~N}$ in the concentric web. Also, the $F_{1 \text { max }}$ was experienced at $t=0.82 \mathrm{~s}$, i.e. at the later stage, than $F_{2 \max }$ was developed, which means that the spiral web behaived as more damped system. This is futher confirmed with the plots of all stresses in the threads of the spiral and concentric webs. As it appears, response of the concentric web is more dramatic, as it can be seen in Fig. 8 .

Saying all this, there are no clear winners in terms of the performance, but based on the preliminary tests, the spiral webs are probably taking the marginal "lead".

\subsection{Non-rotating web is wrapping a co-axial cylindrical disk}

An illustrative case of the collision of the ejected mesh with a cylinder has been simulated and is presented graphically in Fig. 9. The mesh is simulated with $N_{F E}=391$ finite elements with the mesh having $N_{N}=356$ nodes. The FEM analysis is very useful, as enables not only determine the changes in the shape of the mesh, but also enables to monitor the strains/stresses at the different places of the mesh. We were able to simulate the cases of the failure of the spider-like webs, which would be of practical importance for the assessment of the re-usability of the webs for multiple captures.

\subsection{Non-rotating web is wrapping a long cylinder}

A sequence, showing the collision between the flying web and stationary cylinder, is shown in Figs. 10 and 11. This particular study case is characterised with the following parameters: $N_{r}=11, N_{s}=7, R_{0} / R=0.1, R_{f} / R=0.9, L=296.16[\mathrm{~m}], N_{F E}=511$, $N_{N}=446, v=0.5[\mathrm{~m} / \mathrm{s}], \omega=0.005[\mathrm{rad} / \mathrm{s}], M_{e}=20[\mathrm{~kg}], m_{r}=3$ $[\mathrm{kg} / \mathrm{m}], m_{s}=2[\mathrm{~kg} / \mathrm{m}], c_{r}=10[\mathrm{~N} \cdot \mathrm{s} / \mathrm{m}], c_{s}=8[\mathrm{~N} \cdot \mathrm{s} / \mathrm{m}], k_{r}=100$ $[\mathrm{N} / \mathrm{m}], k_{s}=50[\mathrm{~N} / \mathrm{m}]$.
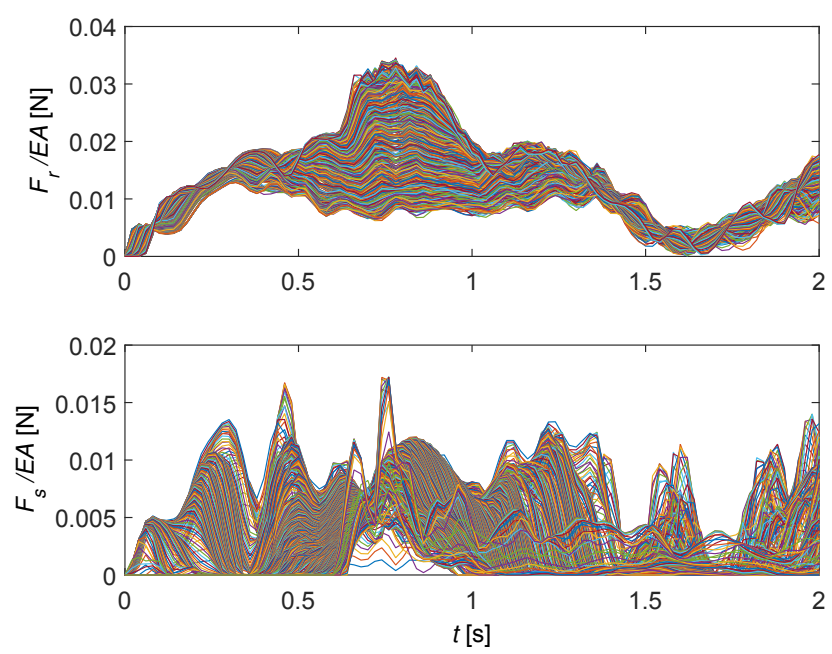

(a)
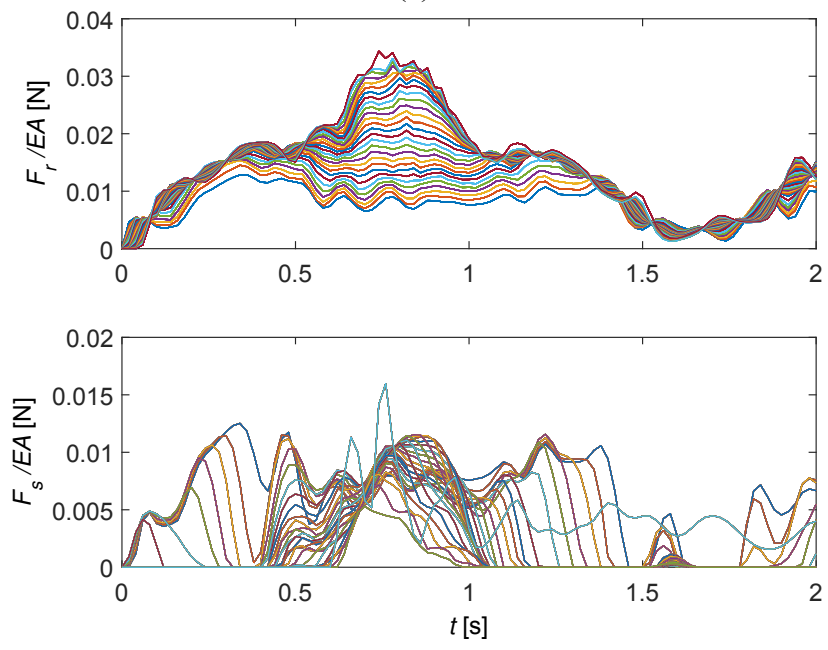

(b)

Fig. 8. Time history of the spiral and radial stresses in all web's threads: (a) Spiral web, (b) Concentric web. 


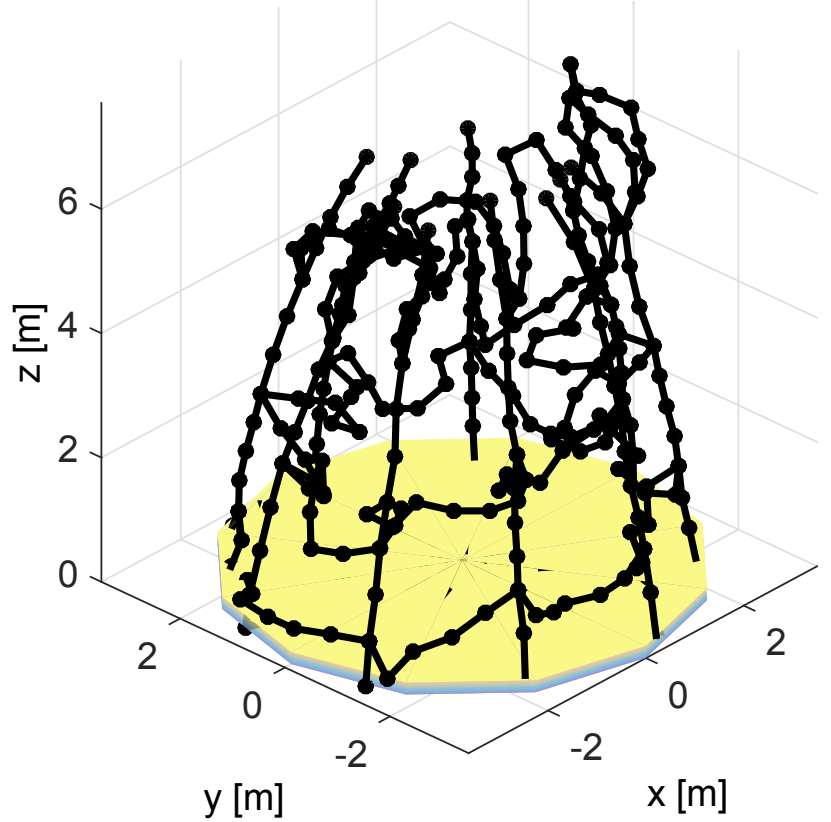

Fig. 9. Ejected non-rotating net captures a flat cylindrical disk.

\subsection{Non-rotating web is wrapping single and multile spherical debris}

A non-symmetrical (3 $\mathrm{m}$ offset) collision of the flying web with $v=0.5 \mathrm{~m} / \mathrm{s}$, hitting a spherical obstacle on its way is shown in Fig. 12. The simulation shows an interesting pattern of wrapping the obstacle. The process can be enhanced with the set of heavy masses attached to the end points of the radii of the mesh.

Numerical simulations of the collisions of the webs with two and three spheres, different in sizes and arbitrarily positioned in space, are presented in Figs. 13 and 14. The efficiency of the capture is strongly dependent upon the size of the web and position of the obstacles. In some cases, when the spread of the obstacles is too wide, the mesh is not capable of completely wrapping the obstacles. However, with the proper proportion of the dimensions of the web and the obstacle, and especially when the collision is close to the symmetrical case, the wrapping could be classified as "absolute", with the obstacle completely covered by the web and further sealed with the radii masses.

\subsection{Comparison of the non-rotating and spin-stabilised webs}

It is of interest to compare performance of the rotating and non rotating meshes. A set of the contrast simulations scenarios is presented in Figs. 15 and 16. It can be observed, that in the considered cases, the non-rotating webs provided a beter embrace (thus, capture) of the targeted obstacles.

\subsection{Influence of the direction of rotation of the spin- stabilised webs}

It is interesting to observe the difference in performance of the same meshes, but rotating in different directions. A set of the contrast simulations scenarios is presented in Figs. 17, and 18 , corresponding to the (a) non-rotating ejected mesh, (b) mesh, rotating in the direction of the web spiral and (c) mesh, rotating against the direction of the web spiral. Undoubtly, the non-rotating mesh, even in the presence of the off-set axial col-
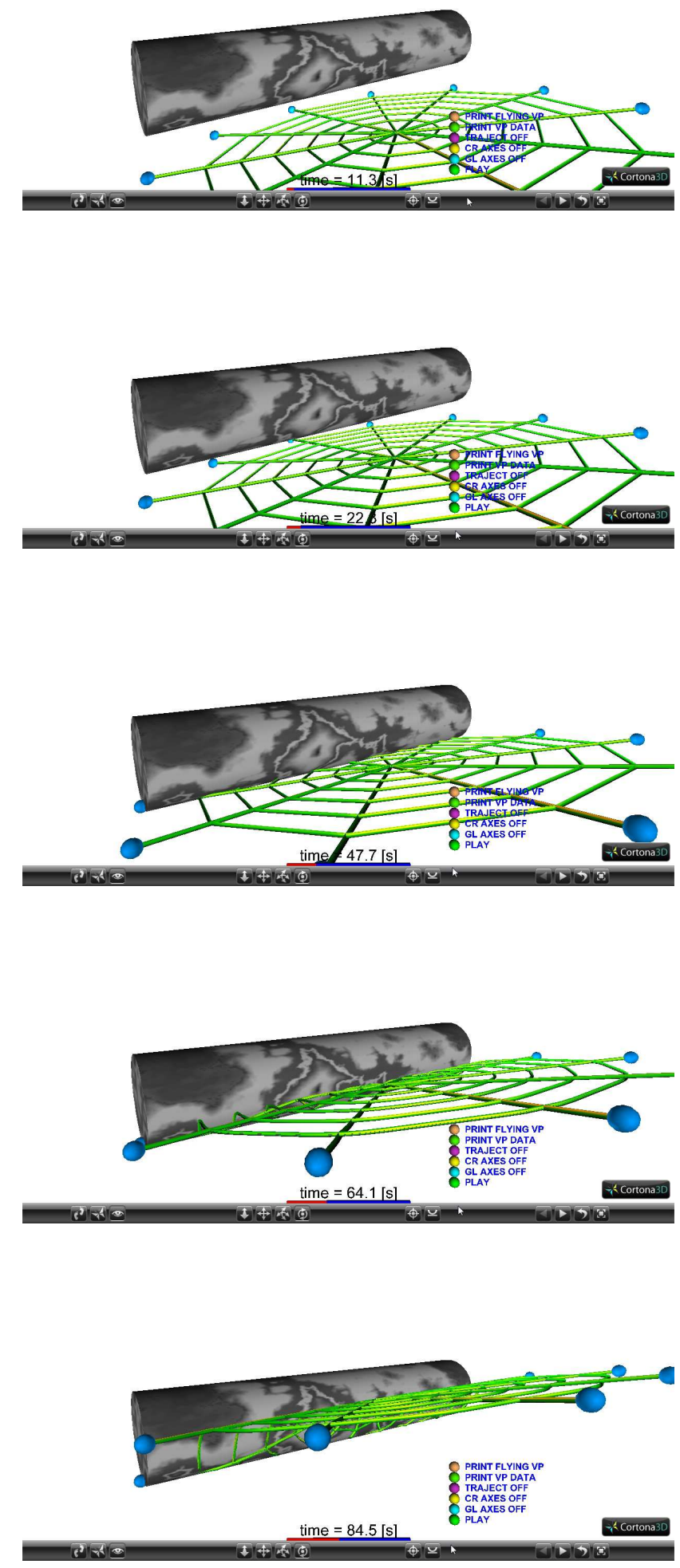

Fig. 10. Time history of the collision of the flying web with a long cylinder.

lision, ensures the best wrapping. As it appears, rotation of the web in the direction of the web's spiral produces higher quality (completeness) of wrapping, at least in the simulated case.

Another interesting observation, based on multiple simulations, which is worthwhile to mention here, is: increase in the 

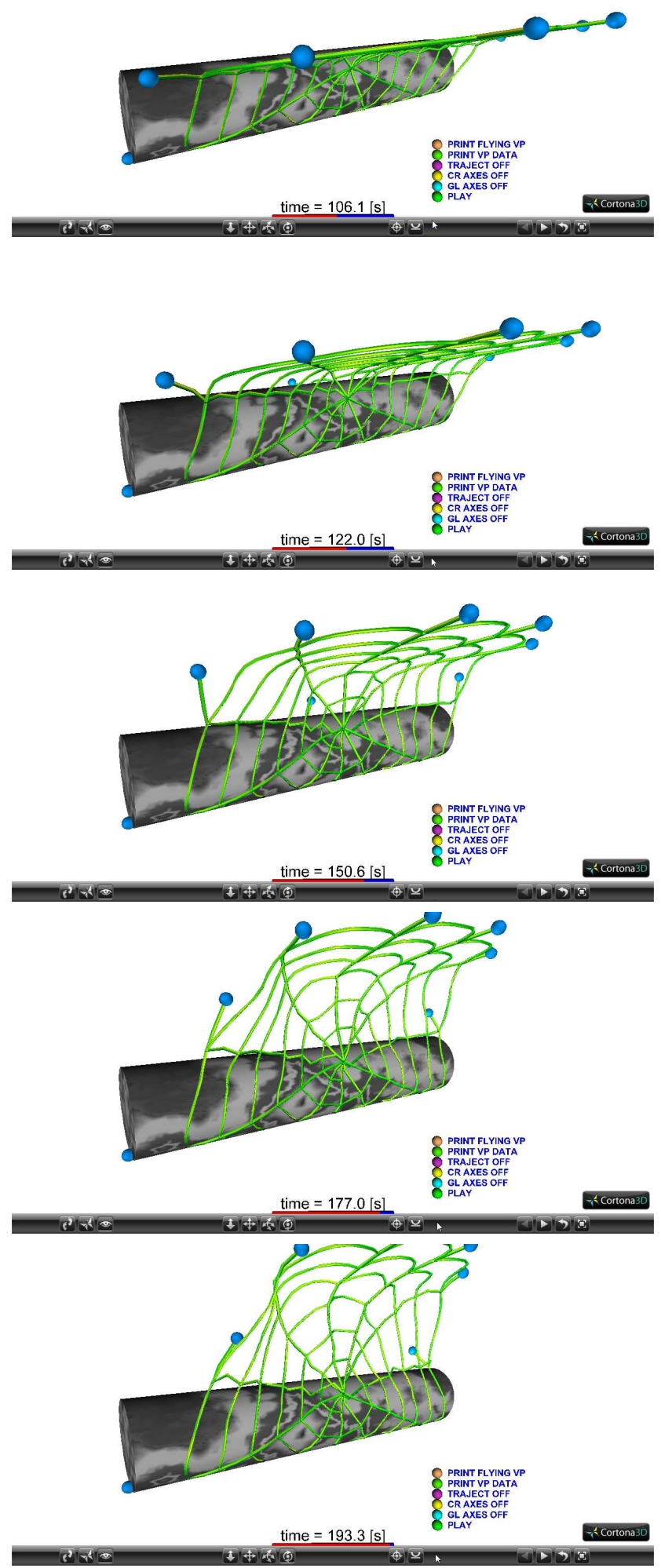

Fig. 11. Time history of the collision of the flying web with a long cylinder (continued).

dimension of the spin-stabilised rotating web does not guarantee a better wrapping.

\subsection{Captures and misses}

In this section we simulate two attempts to capture two spherical objects. In the first case the web's density was not sufficient

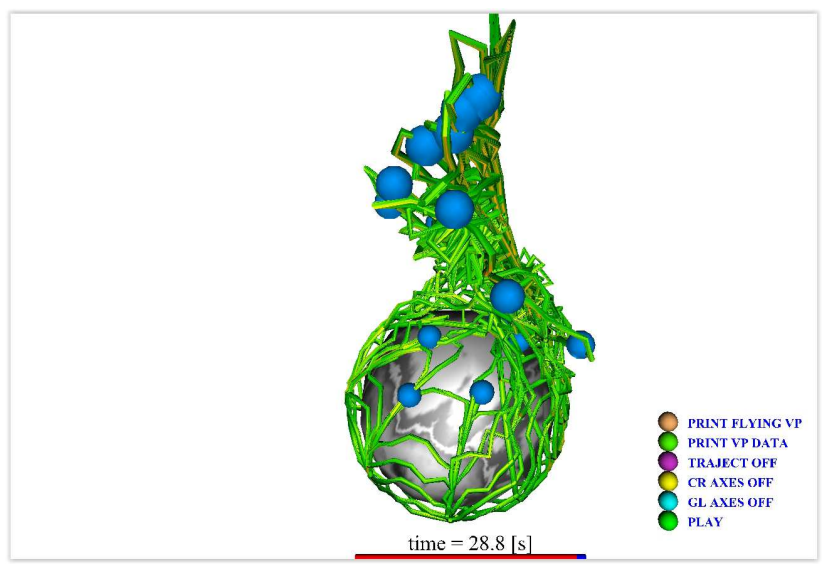

Fig. 12. Non-rotating flying net wrapping an off-set spherical obstacle.

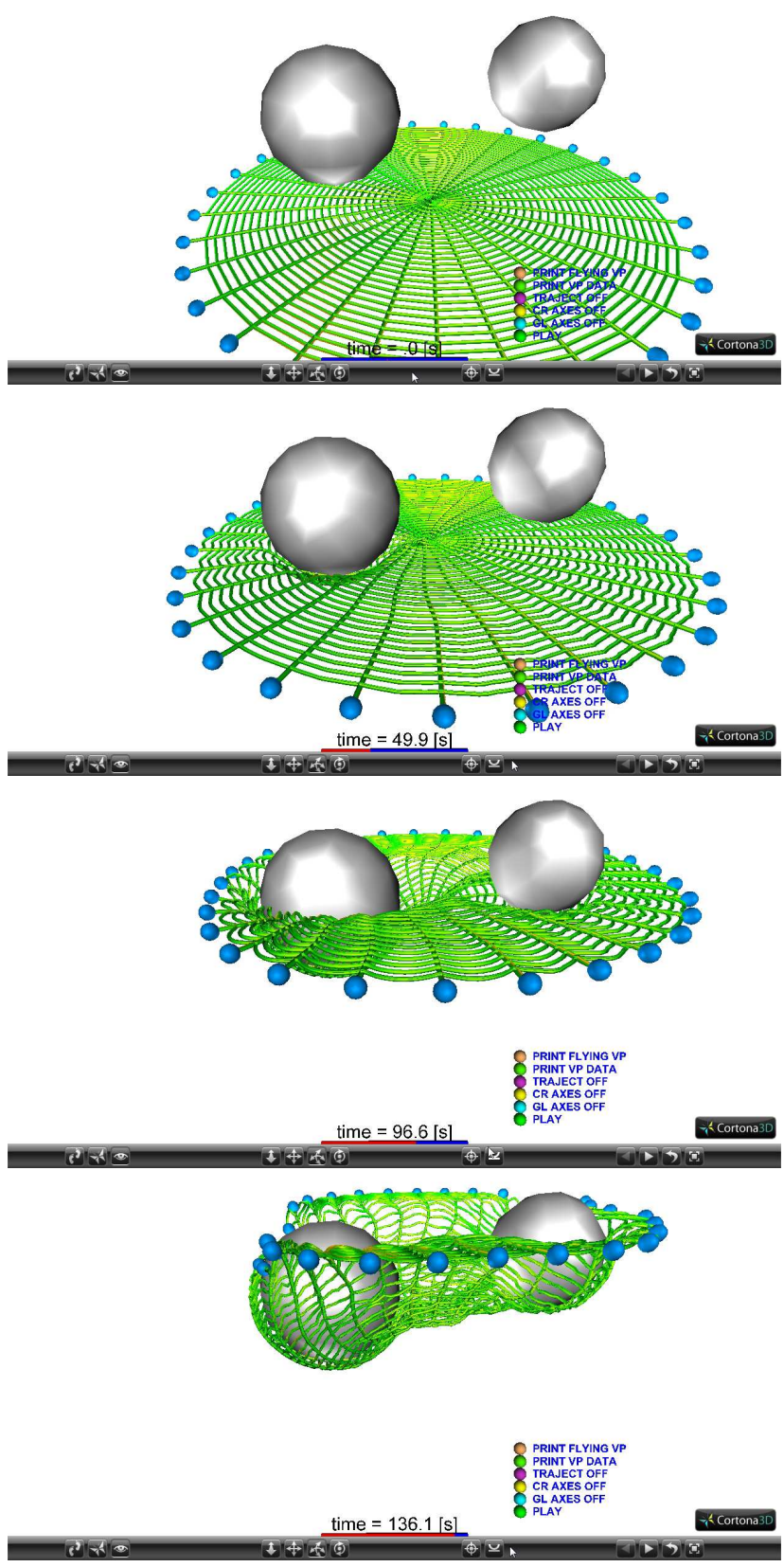

Fig. 13. Collision of the flying web with two spherical debris.

to capture the objects and one of them is slipping through one of 


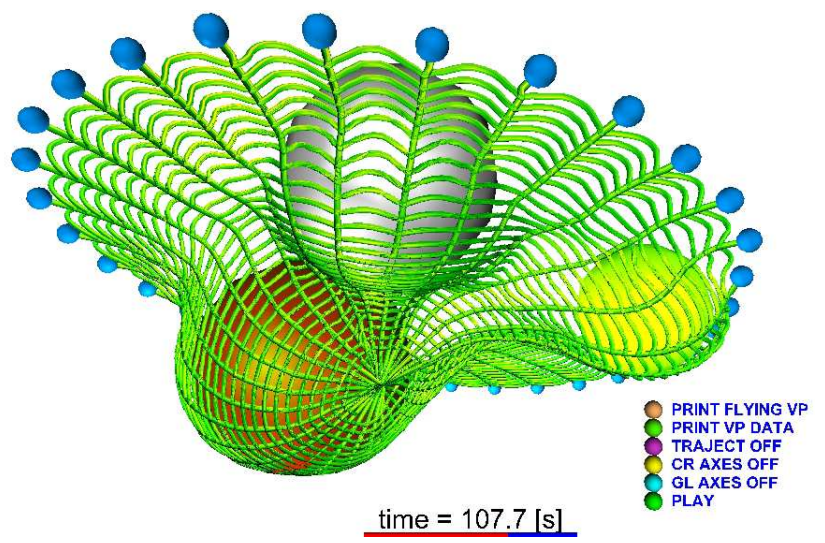

Fig. 14. Flying net is wrapping three spherical debris.

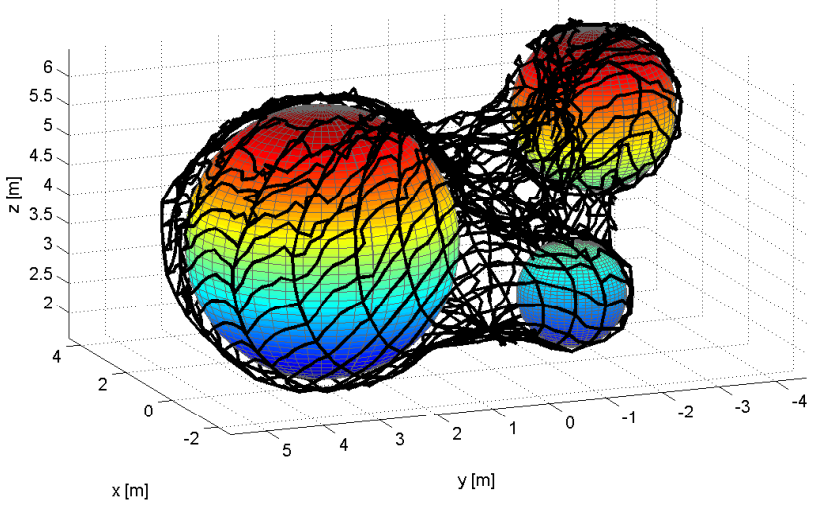

Fig. 15. Ejected non-rotating net captures multiple objects.

the holes in the net. As for the second object, in the same simulation, it has not been captured either, because the interaction of the web with the first sphere caused the web to lump towards its centre, and allowing the second shpere also to "escape". The paramters of this first case are as follows: $N_{r}=15, N_{s}=10$, $R_{0} / R=0.20, R_{f} / R=0.90, L=521.1[\mathrm{~m}], N_{F E}=1,572, N_{N}=1,438$, $v=0.5[\mathrm{~m} / \mathrm{s}], \omega=0[\mathrm{rad} / \mathrm{s}] . c_{r}=10[\mathrm{~N} \cdot \mathrm{s} / \mathrm{m}], c_{s}=8[\mathrm{~N} \cdot \mathrm{s} / \mathrm{m}], k_{r} / m_{r}$ $=330[\mathrm{~N} / \mathrm{m}], k_{s} / m_{s}=250[\mathrm{~N} / \mathrm{m}]$. Results of the simulation are illustrated in Figs. 19-21 with the screen snap-shots from the computer during the animation in Virtual Reality.

A contrast case, where both spheres were successfully captured, is presented in Fig. 22.

\section{Conclusion}

This paper is dedicated to the modelling of the biologically inspired webs, closely replicating the geometry of the spider orb-webs. We implemented the Finite Element method into MATLAB programs, which enablied us to solve various nonlinear study cases, with the emphasis on dynamic of the objects captures and collisions. In particular, in this study we successfully simulated and visualised in Virtual Reality the following scenarios: (a) collision of the ejected webs with the single and multiple obstacles (representing the space debris); (b) collision of the debris with the stationary webs; (c) significant impacts, leading to the failure/rupture of the web. It was possible to predict the cases when the web would not be adequate for capturing the targeted objects. In relation to this, we were able to show via the simulations, that the debris could slip through the

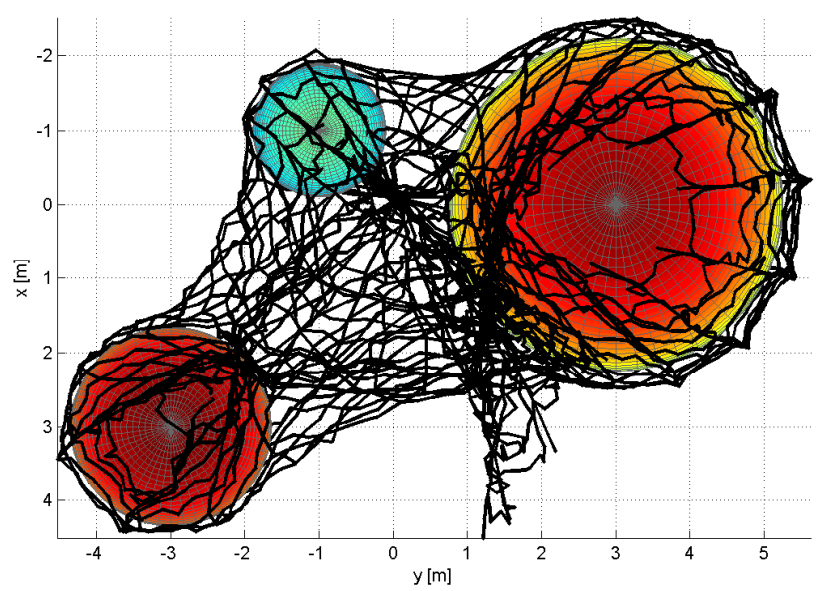

(a)

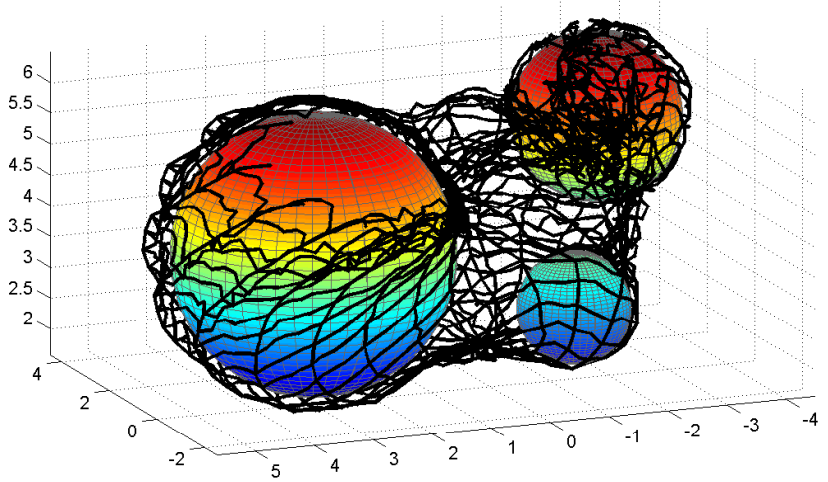

(b)

Fig. 16. Ejected spin-stabilised net captures multiple objects: (a) 3D view, (b) Orthogonal view.

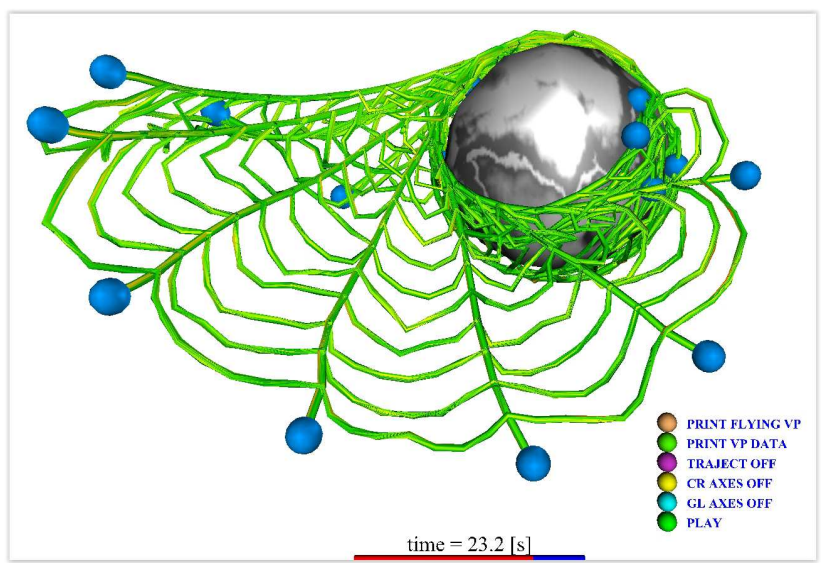

Fig. 17. Flying net, rotating in the direction of the spiral and wrapping an off-set spherical obstacle.

holes in the web, if the geometric parameters of the webs are not carefully selected. In all cases we were able to assess the dynamical parameters of the system and observe the influence of various operational parameters on the dynamics of the postcollision processes. Using the developed simulation platform, it has been demonstrated that the wrapping of the obstacles, associated with space debris, is strongly dependent upon the geometry of the obstacles, their number, relative on-going speed of the web, rotational angular velocity of the spin-stabilised web and the direction of its spin. It has been observed that the rotating webs are not wrapping targeted objects, as non-rotating webs. 


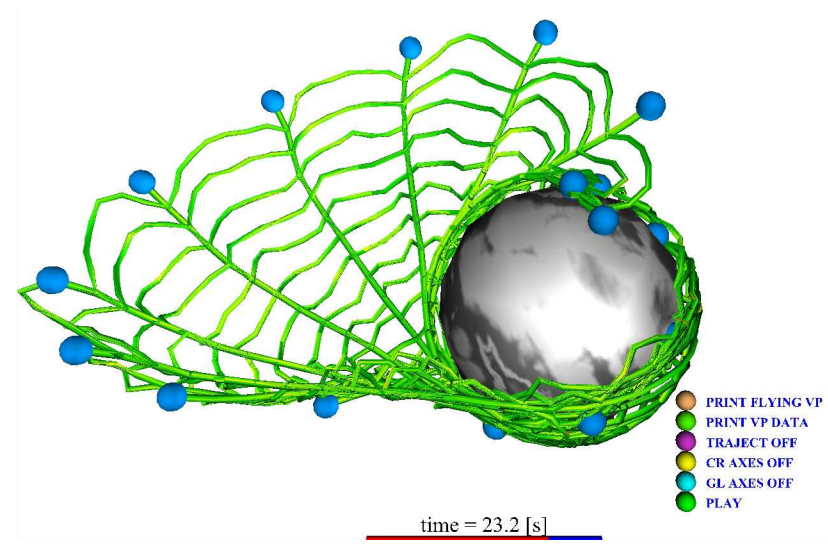

Fig. 18. Flying net, rotating against the direction of the spiral and wrapping an off-set spherical obstacle.

Also, in the limited study cases we were able to observe slightly better strength performance of the spiral webs, as compared to the radial webs. However, this aspect requires more analysis and the authors are dedicated to continue research in the field to address this observation in more detail.

\section{References}

1) http://www.nasa.gov/mission_pages/station/news/orbital_debris.html (cited on Nov. 5, 2015).

2) Wormnes, K., et al.: ESA Technologies for Space Debris Remediation, Proceedings of the 6th IAASS Conference: Safety is Not an Option, 2013, pp. 3-4.

3) Kjetil, W., Le Letty, R., Summerer, L., Schonenborg, R., DuboisMatra, O., Luraschi, E., Cropp, A., Krag, H. and Delaval, J.: ESA Technologies for Space Debris Remediation, Proceedings of the 6th IAASS Conference: Safety is Not an Option, 2013, pp. 3-4.

4) Blanksby, C. and Trivailo, P.: Assessment of Actuation Methods for Manipulating Tip Position of Long Tethers (Invited Paper), Space Technology: Space Engineering, Telecommunication, System Engineering and Control, (Lister Science, Great Britain), 20 (2000), pp. 31-39.

5) Williams, P.: Nonlinear Control and Applications of Tethered Space Systems, PhD Thesis. RMIT University. (2004). - 445 pp.

6) Nakasuka, S., Aoki, T., Ikeda, I., et al.: "Furoshiki Satellite" - A Large Membrane Structure as a Novel Space System, Acta Astronautica, 48 (2001), pp. 461-468.

7) Benvenuto, R. and Lavagna, M. R.: Flexible Capture Devices for Medium to Large Debris Active Removal: Simulations Results to Drive Experiments, Proceedings of the 12th Symposium on Advanced Space Technologies in Automation and Robotics. (2013).

8) Ruggiero, A., Pergola, P. and Andrenucci, M.: Small Electric Propulsion Platform for Active Space Debris Removal. Paper IEPC2013-217, Proceedings of The 33st International Electric Propulsion Conference, The George Washington University, USA, October 6-10, 2013, $12 \mathrm{pp}$.

9) First Mission of the Tethered Satellite System, NASA Technical Memorandum NASA-TM-107955, US Government Printing Office, 1992-324-999, (1992). - $41 \mathrm{pp}$

10) Nishida, S. and Kawamoto, S.: Dynamical Simulations for Space Debris Capture, Proceedings of the SICE Annual Conference (SICE), (2011), pp. 2283-2286, 13-18 Sept. 2011.

11) Pearson, J., Levin, E., Oldson, J. and Carroll, J.: ElectroDynamic Debris Eliminator (EDDE): Design, Operation, and Ground Support. STAR Technology And Research Inc., Mount Pleasant, SC, 2010. -9 pp.

12) Blanskby, C.: Tracking Control of Space Tethers. PhD Thesis. RMIT University. (2001). -213 pp.

13) Trivailo, P.M., Blanksby, C. and Keramati Nigjeh, B.: Advances in Space Tether Applications, Proceedings of the NSSA Space Science

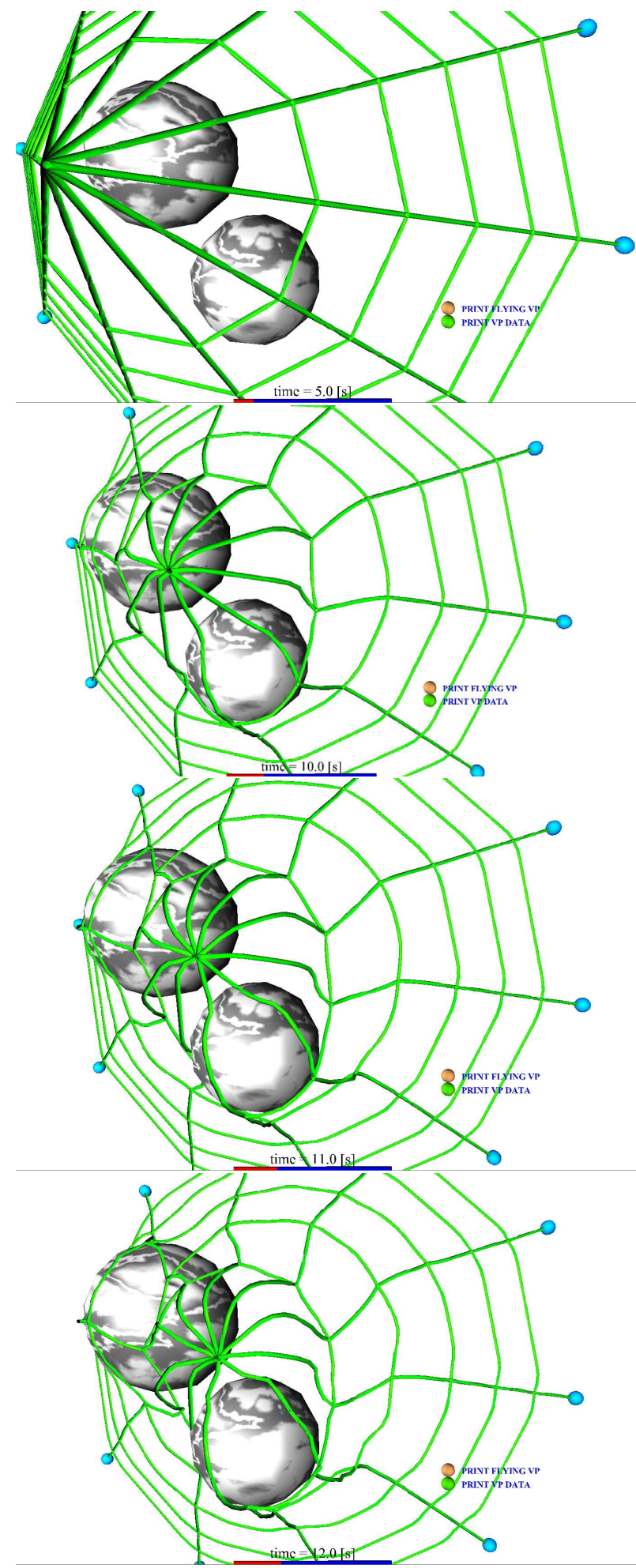

Fig. 19. Unsuccessful attempt to capture two spherical objects.

Workshop, University of Sydney, 18-20 July, 2001. (2001). - 20 pp.

14) Keramati Nigjeh, B.: Design Analysis of a Space Capturing Systems Using Tether Technology, PhD Thesis. RMIT University. (2002). $226 \mathrm{pp}$.

15) Williams, P., Blanksby, C., Trivailo, P., Fujii, H.A. and Kojima, H.: Space Tether Technology for Payload Capture, Space Utilization Research, 19 (2003), Proceedings of the Nineteenth Space Utilization Symposium, January 20-21, 2003, Institute of Space and Astronautical Science, Sagamihara, Kanagawa, Japan, pp. 137-140.

16) Carroll, J. A.: Space transport development using orbital debris. Final Report on NIAC Phase I, Research Grant 07600-087 (2002), 

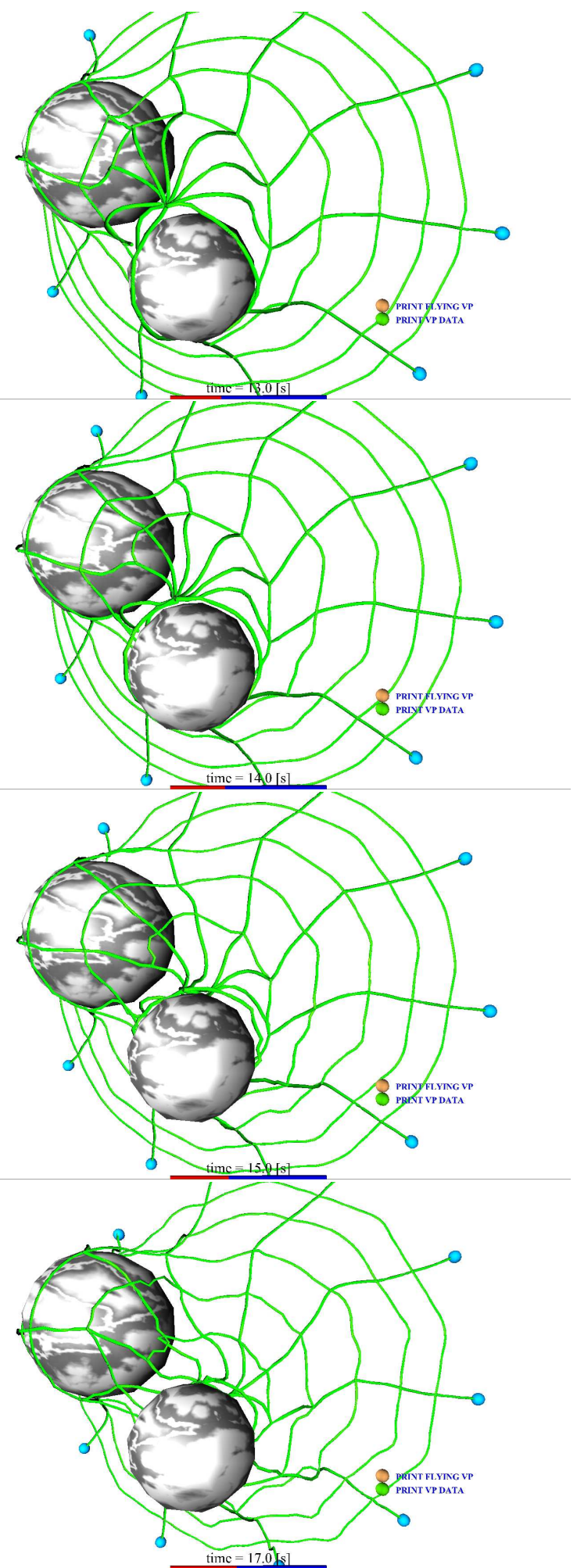

Fig. 20. Unsuccessful attempt to capture two spherical objects (continued).

\section{pp. 1-43.}

17) Yu, Y., Baoyin, H.-X. and Li, J.-F.: Dynamic Modelling and Analysis of Space Webs, Science China Physics, Mechanics and Astronomy, 54 (2011), pp. 783-791.

18) Guang, Z., Qiu, Y., Liang, B. and Li, C.: On-orbit Capture with Flexible Tether-Net System, Acta Astronautica, 65 (2009), pp. 613623.

19) Aoyanagi, Y. and Okumura, K.: Simple Model for the Mechanics of Spider Webs, Physical Review Letters. PRL 104, 038102 (2010,
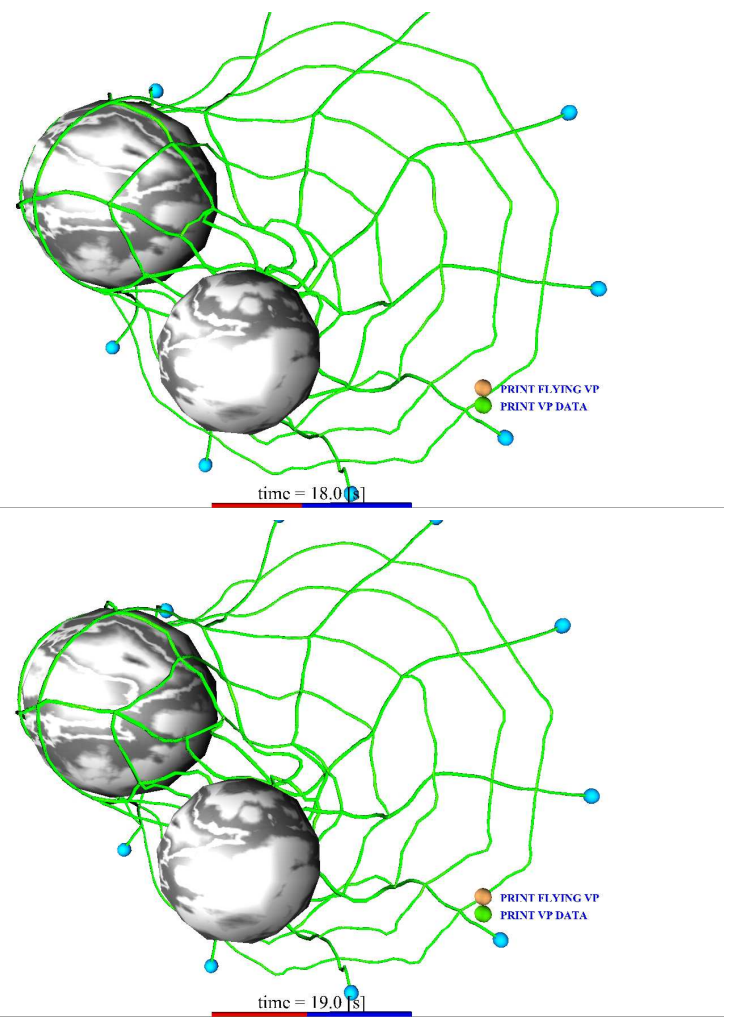

Fig. 21. Unsuccessful attempt to capture two spherical objects (continued).

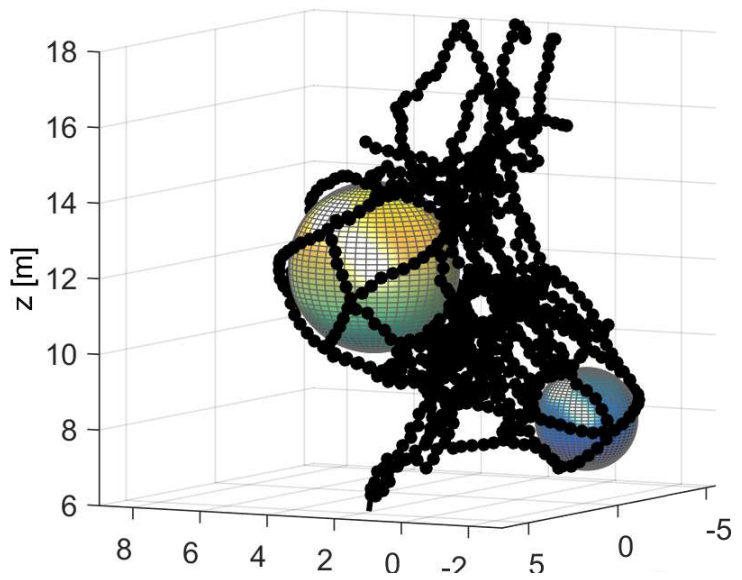

Fig. 22. Successful capture of two spherical objects.

week ending 22 January), pp. 038102(1)-038102(4).

20) Cranford, S.W., Tarakanova, A., Pugno, N.M. and Buehler, M.J.: Nonlinear Material Behaviour of Spider Silk Yields Robust Webs, Nature, 482 (2012), pp. 72-78.

21) Vollrath, F.V., Downes, M. and Krackow S.: Design Variability in Web Geometry of an Orb-Weaving Spider, Physiology $\mathcal{E}$ Behavior, 62 (1997), pp. 735-743.

22) Schmitt, W., Walter, M., Comba, J.L.D.: Realistic Modeling of SelfAdapted Spider Orb-Webs in Real-Time, Proceedings of the 2013 XXVI Conference on Graphics, Patterns and Images, SIBGRAPI 2013, Arequipa, Peru, August 5-8, 2013, IEEE Computer Society, pp. 288-295.

23) http://www.loc.gov/rr/scitech/mysteries/spiderweb.html (cited on May, 05 2015).

24) Sensenig, A.T., Lorentz, K.A., Kelly, S. and Blackledge, A.: Spider Orb Webs Rely on Radial Threads to Absorb Prey Kinetic Energy, Journal of the Royal Society Interface, 9 (2012) , pp .1880-1891.

25) Zheng, L., Behrooz, M., Li, R., Wang, X. and Gordaninejad, F.: Performance of a Bio-Inspired Spider Web. -9 pp. 